\title{
Effet des cordons pierreux sur l'activité biologique d'un sol ferrugineux tropical lessivé (Province du Kouritenga au Burkina Faso)
}

\author{
Sabine Marie Flore DOAMBA ${ }^{1}$, Hassan Bismarck NACRO ${ }^{1 *}$, Antoine SANON ${ }^{2}$ et \\ Michel SEDOGO ${ }^{3}$ \\ ${ }^{1}$ Institut du Développement Rural, Laboratoire d'Etude de Recherche sur la Fertilité des Sols (LERF), \\ Université Polytechnique de Bobo-Dioulasso, 01 BP 1091 Bobo-Dioulasso, Burkina Faso. \\ ${ }^{2}$ UFR-SVT, Université de Ouagadougou, 03 BP 7021 Ouagadougou, Burkina Faso. \\ ${ }^{3}$ Institut de l'Environnement et de Recherches Agricoles, 04 BP 8645 Ouagadougou, Burkina Faso. \\ *Auteur correspondant : nacrohb@yahoo.fr
}

\section{RESUME}

Afin de comprendre le mécanisme de restauration des sols dégradés, l'effet des cordons pierreux sur la macrofaune (méthode TSBF), les teneurs en carbone organique (méthode de Walkley et Black) et en azote (Kjeldahl) et sur l'activité biologique du sol (mesure du dégagement du $\mathrm{CO}_{2}$ ) a été évalué. Les résultats montrent que les cordons pierreux ont un effet positif sur la macrofaune du sol : en effet, 208 individus ont été recensés dans le champ aménagé en cordons pierreux, contre seulement 32 individus dans le champ témoin. Concernant la diversité, 13 familles ont été recensées dans le champ aménagé, contre 6 familles dans témoin. La teneur en éléments fins varie de $29 \%$ pour le sol témoin, à $67 \%$ pour le sol aménagé. Les teneurs en carbone et en azote total passent respectivement de $4,9 \mathrm{~g} \mathrm{C} / \mathrm{kg}$ sol pour le sol témoin à $7,5 \mathrm{~g} \mathrm{C} / \mathrm{kg}$ sol pour le sol sous cordons pierreux, et de $0,48 \mathrm{~g} \mathrm{~N} / \mathrm{kg}$ de sol à $0,69 \mathrm{~g} \mathrm{~N} / \mathrm{kg}$ sol. On observe également, une amélioration du potentiel de minéralisation du carbone. Ces résultats montrent très clairement que les cordons pierreux ont un effet positif sur l'amélioration des paramètres de la fertilité des sols du sahel.

(C) 2011 International Formulae Group. All rights reserved.

Mots clés : Cordon pierreux, fertilité, macrofaune du sol, sol ferrugineux tropical lessivé, Burkina Faso.

\section{INTRODUCTION}

La plupart des sols de l'Afrique de l'Ouest se distinguent par une mauvaise stabilité structurale des horizons superficiels, leur richesse en limons et sables fins, leur faible teneur en argile, en matière organique (inférieurs à $3 \%$ sous végétation, et $0,7 \%$ sous cultures), et la faible réserve minérale (Pieri, 1989; Saidani, 1995). Ces facteurs, auxquels il faut ajouter la faiblesse de la production primaire, notamment hypogée et les conditions pédoclimatiques entraînant une dégradation rapide de la matière organique, ne font qu'aggraver le statut déjà faible de la fertilité physique et chimique des sols de cette région.

Environ $65 \%$ des terres agricoles ont été affectés par les phénomènes de dégradation au cours des 40 dernières années (Scherr, 1999; Katyal et Vlek, 2000; Bationo et al., 2007). Cette dégradation entraîne une baisse de la fertilité chimique, physique et biologique des sols, constituant une menace grave pour la sécurité alimentaire. Par 
exemple, en Afrique sub-saharienne, on estime que les pertes en azote, phosphore et potassium se situent entre 15 et $71 \mathrm{~kg} / \mathrm{ha}$, du fait de l'érosion, de l'exportation des résidus de culture et du faible niveau de fertilisation des sols (Stoorvogel et Smaling, 1990; Henao et Baanante, 2006).

Parmi les causes physiques de la dégradation des sols, on peut citer l'érosion, qui est responsable de la dégradation de $72 \%$ des terres arables de l'Afrique et $31 \%$ des terres pastorales. Les terres perdues par érosion sont 2,5 fois plus riches en éléments nutritifs que le sol restant (Bationo et al., 1998). Au Burkina Faso, on estime qu'environ $24 \%$ des terres arables sont fortement dégradées et que $31 \%$ de la pluviosité annuelle est perdue par ruissellement (INERA, 2000). Cette situation se traduit par une dégradation continue des terres par suite de la surexploitation du sol et de la disparition progressive du couvert végétal, entraînant la formation de glacis dénudés et dégradés (Roose et al., 1993).

Face à cette dégradation prononcée des sols, les populations ont développé des actions de défense et restauration des sols (DRS) et des actions de conservation des eaux et des sols (CES) par la construction d'ouvrages anti-érosifs tels que les digues filtrantes, les demi-lunes, les diguettes, les haies vives, le paillage, le zaï et les cordons pierreux. Ces derniers sont des barrières physiques placées perpendiculairement à la pente pour freiner le ruissellement et réduire l'érosion. Il s'en suit une augmentation de l'infiltration de l'eau dans le sol, et la sédimentation des particules (sables, terre fine, matière organique) à l'amont de la diguette (Dugué et al., 1993; Hien, 1995). Cette technique permet à la fois de restaurer les paysages naturels (Sangaré, 2002; Doamba, 2007) et la fertilité des sols, et donc, d'augmenter la quantité et la qualité de la production agricole (Kambou et Zougmoré, 1995).

Toutefois, si de nombreux travaux existent sur l'effet de ces ouvrages sur les caractéristiques physico-chimiques des sols dégradés, très peu d'études se sont intéressées à la quantification de l'activité biologique dans ces sols. Pourtant, la dégradation biologique constitue une des formes de dégradation des terres, de baisse des capacités de production due à l'altération des caractéristiques des sols. Cette dégradation se traduit par la baisse des activités microbiennes de minéralisation de la matière organique, mais aussi par la réduction de l'activité de la macrofaune du sol consécutive à la dégradation physique des sols. L'objectif de ces travaux est donc d'étudier l'impact de l'aménagement en cordons pierreux sur l'évolution de la fertilité biologique des sols, au travers de l'évaluation de la teneur en carbone et azote, du potentiel respiratoire, de l'abondance et de la diversité de la faune du sol.

\section{MATERIEL ET METHODES \\ Site d'étude}

La zone d'étude est située à Koupéla (province du Kouritenga), à $140 \mathrm{~km}$ à l'Est de la ville de Ouagadougou. La zone appartient au domaine phytogéographique Nord-sahélien (Fontès et Guinko, 1995). Elle est caractérisée par l'alternance de deux saisons fortement contrastées: une saison des pluies, de mai à octobre et une saison sèche de novembre à avril. Les travaux se sont déroulés dans le village de Kampelzougou $\left(12^{\circ} 25734 \mathrm{~N}, 00^{\circ}\right.$ 26689 0), village situé à $50 \mathrm{~km}$ de la ville de Koupéla. Les champs concernés par notre étude ont été mis en culture depuis plusieurs années, avant d'être aménagés en cordons pierreux en 2006 par un paysan innovateur. La pluviosité annuelle du site varie de 750 à 1000 $\mathrm{mm}$. Les sols sont à dominance ferrugineux tropicaux lessivés, et surtout caractérisés par leur faible teneur en azote et en phosphore.

\section{Fouille et inventaire de la macrofaune du sol}

L'estimation de la macrofaune du sol a été faite selon la technique des carrés avec fouille du sol par tri manuel selon la méthode TSBF (Tropical Soil Biology and Fertility) (Anderson et Ingram, 1989). La fouille du sol s'effectue à la houe, après avoir délimité un 
carré de $25 \mathrm{~cm} \times 25 \mathrm{~cm}$ de côté. Des opérateurs creusent simultanément une tranchée autour de ce carré, jusqu'à $30 \mathrm{~cm}$ de profondeur, afin de limiter autant que possible la fuite des insectes. Le bloc de terre ainsi délimité est ensuite fractionné et émietté à la main. Le tri s'effectue à l'aide de pinces. Les individus de la macrofaune ainsi récoltés sont conservés dans des piluliers remplis d'alcool à $70 \%$. La macrofaune du sol, soigneusement récoltée, a été observée et dénombrée au laboratoire. Plusieurs clés de détermination ont été utilisées (Bland et Jacques, 1987; Villiers, 1997 ; CIRAD et CNEARC, 1998 ; Delvare et Aberlenc, 1999).

\section{Mesures des paramètres physico-chimiques du sol}

Les parcelles aménagées en cordons pierreux ont été subdivisées en trois bandes de pierres suivant la pente (Figure 1). Au total, 30 échantillons de sol ont été prélevés (horizon 0-20 cm) à l'intérieur des bandes, soit 9 échantillons par bande de cordons pierreux. Le sol a également été prélevé dans un champ témoin non soumis à la technique des cordons pierreux. Tous les échantillons de sol ont été tamisés à $2 \mathrm{~mm}$, puis conservés séparément en vue des analyses physicochimiques. Les prélèvements ont été effectués en mai 2008.

La répartition de la matière organique en fraction organo-minérale a été déterminée selon la méthode décrite par Feller (1979). Trois fractions ont été obtenues: fraction organo-minérale $\quad(0-50 \mu \mathrm{m}), \quad$ fraction organique associée aux sables fins (50- 200 $\mu \mathrm{m})$, fraction organique associée aux sables grossiers (200-2 $000 \mu \mathrm{m})$. Le dosage du carbone total et de l'azote total de chaque échantillon de sol a été effectué respectivement par la méthode de Walkley et Black (1934) et par celle de Kjeldahl (Bremner, 1965).

\section{Mesure de la respiration du sol}

Le principe est basé sur la mesure du dégagement de $\mathrm{CO}_{2}$ par l'échantillon de sol incubé dans une enceinte close. Le $\mathrm{CO}_{2}$ dégagé est piégé dans une solution de $\mathrm{NaOH}$ puis titré par $\mathrm{HCl}$ (Dommergue, 1960; Bachelier, 1973; Tiessen et Moir, 1993). Le test a consisté à mesurer, la quantité de $\mathrm{CO}_{2}$ dégagé par des échantillons de sols incubés, préalablement humidifiés aux $2 / 3$ de leur capacité maximale de rétention. Le $\mathrm{CO}_{2}$ piégé par la soude est précipité par $3 \mathrm{ml}$ d'une solution de chlorure de baryum (3\%) et dosé par de l'acide chlorhydrique $(\mathrm{HCl} 0,1 \mathrm{~N})$ en présence de la phénophtaléine comme indicateur coloré. Le dosage a été effectué quotidiennement pendant les 7 premiers jours et tous les 2 jours du $8^{\text {ème }}$ au $14^{\text {ème }}$ jour d'incubation.

\section{Analyse des données}

Les données ont été soumises à une analyse de variance (ANOVA) avec le logiciel XLSTAT 7,5. Les moyennes ont été séparées au seuil de $5 \%$ selon le test de NewmanKeuls. Pour l'inventaire de la macrofaune du sol, les données sont obtenues après des calculs de certains paramètres comme l'indice de Shannon-Weaver (H') et celui de l'Equitabilité (E). Ce sont :

$\mathrm{H}^{\prime}=\Sigma$ Pi $\log 2 \mathrm{Pi}$, Pi étant la probabilité de rencontrer un taxon i sur une parcelle; $\mathrm{E}=\mathrm{H}^{\prime} / \log _{2}(\mathrm{~S}) ; \mathrm{S}$ étant le nombre total des taxons rencontrés sur la parcelle.

\section{RESULTATS \\ Variation de la macrofaune du sol}

Au total, 208 individus ont été recensés dans le champ aménagé en cordons pierreux, contre seulement 32 individus dans le champ témoin (Tableau 1). Du point de vue de la diversité spécifique, le nombre de familles rencontrées varie du simple au double: 13 familles recensées dans le champ aménagé en cordons pierreux, contre 6 familles dans le champ témoin (Tableau 1). Les résultats montrent donc que les cordons pierreux ont un effet positif sur les populations de macrofaune du sol. C'est ce que confirme l'indice de diversité de Shannon-Weaver, qui traduit la diversité du peuplement (Tableau 2). En effet, l'indice de diversité, calculé pour le champ aménagé en cordon pierreux, est jusqu'à trois 
fois plus élevé que celui du champ témoin : 0,54 contre 1,25 à 1,76 . L'indice d'équitabilité traduit le rapport entre la diversité observée et la diversité théorique maximale qui correspondrait à une équirépartition des effectifs dans le peuplement. Sa valeur varie de 0,09 pour le champ témoin, à 0,3 pour les champs aménagés (Tableau 2). La pose des cordons pierreux induirait donc une meilleure répartition des effectifs de la macrofaune du sol. De plus, on note la présence d'un nombre plus important de Formicidae (110), Lumbricidae (6), Termitidae (74) dans la parcelle aménagée. Ces familles appartiennent à la macrofaune du sol considérée comme des ingénieurs du sol (Jones et al., 1994).
Variation des paramètres physicochimiques et de l'activité microbienne du sol

La teneur en éléments fins $(0-50 \mu \mathrm{m})$ varie de $29 \%$ pour le sol témoin, à $67 \%$ pour le sol sous cordons pierreux (Tableau 3). On observe que l'aménagement des champs en cordons pierreux entraîne également une amélioration de la teneur du sol en carbone organique : de 4,9 g/kg sol à 7,5 g/ $/ \mathrm{kg}$ sol. Il en est de même pour la teneur en azote total qui varie de $0,48 \mathrm{~g} / \mathrm{kg}$ de sol à $0,69 \mathrm{~g} / \mathrm{kg}$ sol (Tableau 3).

L'activité microbienne de minéralisation du carbone est présentée dans la Figure 3. Les plus faibles dégagements journaliers de $\mathrm{CO}_{2}$ sont enregistrés pour le sol témoin (211 $\mathrm{mg} \mathrm{CO} / \mathrm{kg}$ sol) et pour le sol prélevé en bas de pente (197 $\mathrm{mg} \mathrm{CO}_{2} / \mathrm{kg}$ sol). Par contre, l'activité biologique est plus intense en haut (361 mg CO $2 / \mathrm{kg}$ sol) et en milieu de pente (255 mg CO $2 / \mathrm{kg} \mathrm{sol}$ ).

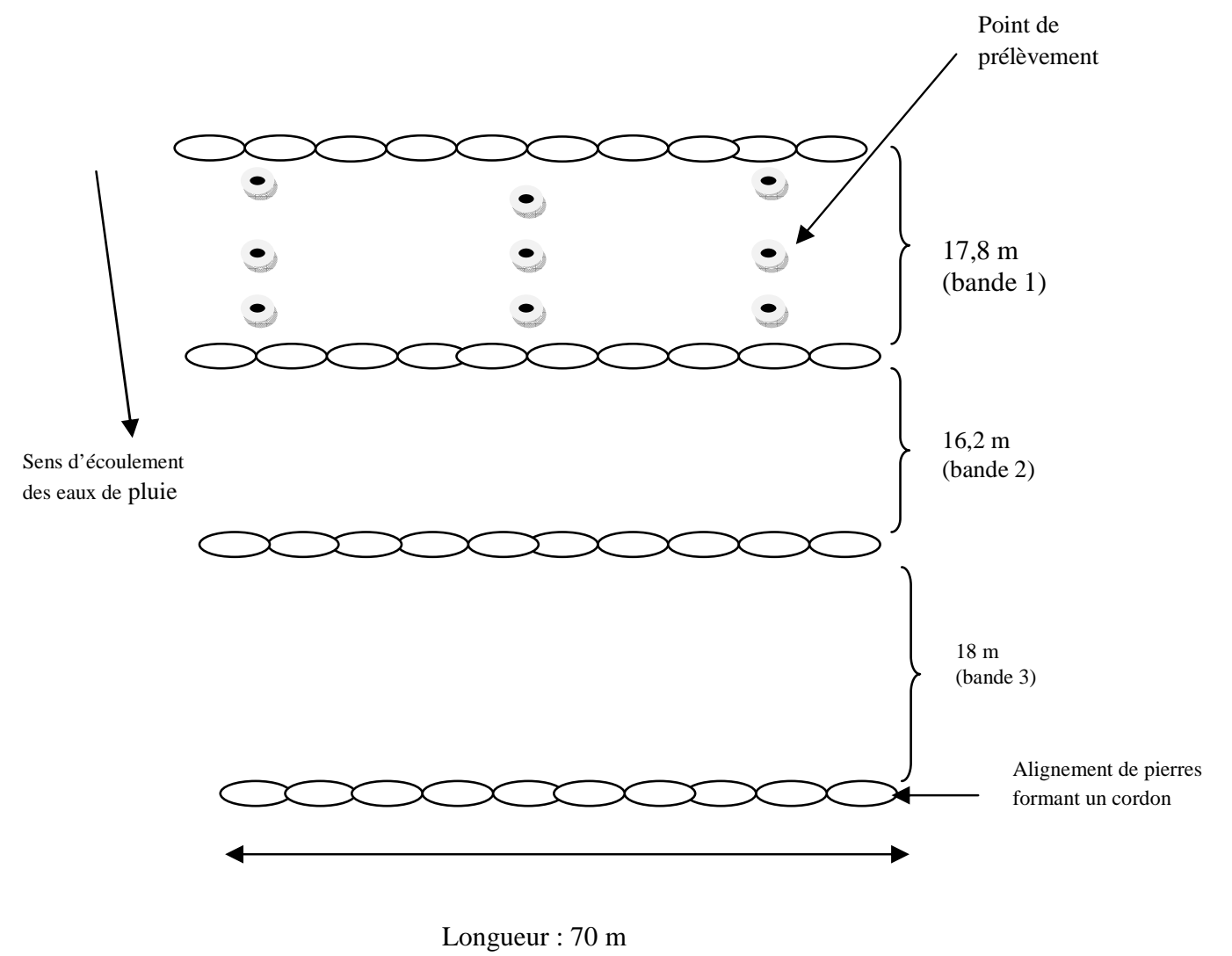

Figure 1: Parcelle aménagée en cordons pierreux. 


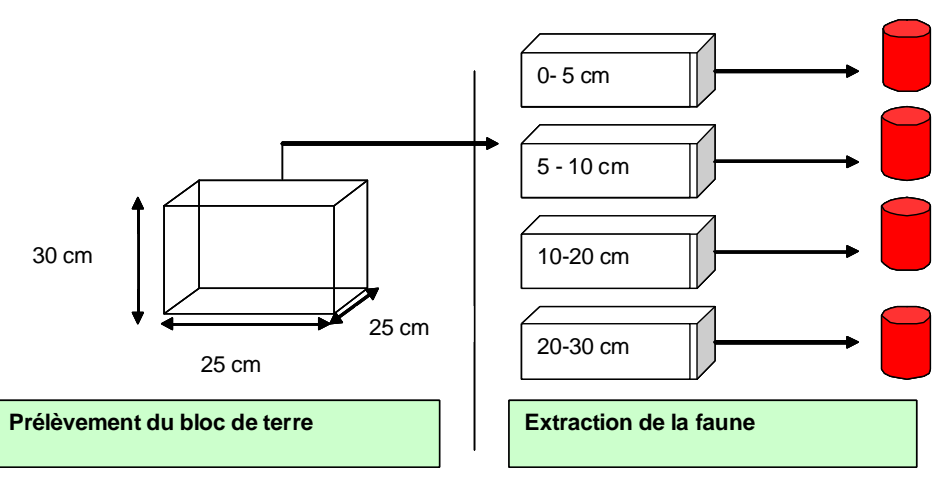

Figure 2: Dispositif de prélèvement de la macrofaune du sol (méthode TSBF).

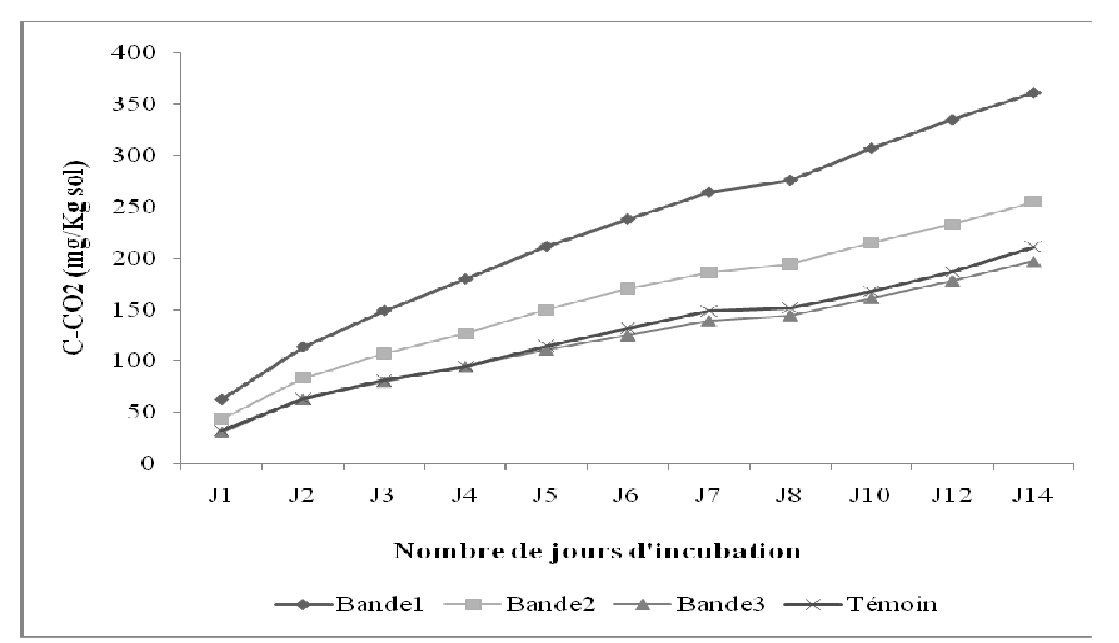

Figure 3: Evolution cumulée du C- $\mathrm{CO}_{2}(\mathrm{mg} / \mathrm{kg}$ sol) dégagé pour chaque traitement (bandes de cordons de pierres et témoin).

Tableau 1: Matrice d'abondance des familles de macrofaune répertoriées sur les parcelles étudiées.

\begin{tabular}{llcc}
\hline \multicolumn{1}{c}{ Ordre } & Familles & $\begin{array}{c}\text { Parcelles } \\
\text { aménagées }\end{array}$ & $\begin{array}{c}\text { Parcelle } \\
\text { témoin }\end{array}$ \\
\hline Aranea & Araneidae & 1 & 0 \\
& Divers Aranea & 3 & 0 \\
\hline Hyménoptères & Formicidae & 110 & 21 \\
Orthptères & Gryllidae & 5 & 0 \\
Haplotaxida & Lumbricidae & 6 & 1 \\
Geophilomorpha & Geophilidae & 3 & 5 \\
Scutigeromorpha & Scutigeridae & 1 & 1 \\
Scorpionida & Scorpionidae & 1 & 0 \\
\hline \multirow{3}{*}{ Coléoptères } & Staphylinidae & 1 & 0 \\
& Tenebrionidae & 1 & 1 \\
\hline Isoptères & Scarabaeidae & 1 & 0 \\
Acariens & Termitidae & 74 & 3 \\
\hline \multicolumn{2}{c}{ (Nombre d'échantillons par traitement $: \mathrm{n}=27$ ) } & 1 & 0
\end{tabular}


Tableau 2: Indices de diversité de la macrofaune du sol.

\begin{tabular}{llcc}
\hline Types de parcelles & Emplacement & $\begin{array}{c}\text { Indice de Shannon } \\
\text { Weatver }\end{array}$ & Equitabilité \\
\hline & $\begin{array}{l}\text { Haut de pente } \\
\text { (Bande 1) }\end{array}$ & 1,76 & 0,30 \\
$\begin{array}{l}\text { Parcelle avec } \\
\text { cordons pierreux }\end{array}$ & $\begin{array}{l}\text { Milieu de pente } \\
\text { (Bande 2) } \\
\text { Bas de pente } \\
\text { (Bande 3) }\end{array}$ & 1,34 & 0,22 \\
\hline Parcelle témoin & - & 0,54 & 0,21 \\
\hline (Nombre d'échantillons par traitement: $\mathrm{n}=9)$ & & 0,09 \\
\hline
\end{tabular}

Tableau 3: Caractéristiques physico-chimiques des sols.

\begin{tabular}{llccccc}
\hline $\begin{array}{l}\text { Types de } \\
\text { Parcelles }\end{array}$ & $\begin{array}{l}\text { Emplacement } \\
\text { en fonction de } \\
\text { la pente }\end{array}$ & $\begin{array}{c}\text { Eléments } \\
\text { fins }\end{array}$ & $\begin{array}{c}\text { C-Total } \\
\text { (g/kg sol) }\end{array}$ & $\begin{array}{c}\text { N-Total } \\
\text { (g/kg sol) }\end{array}$ & pHeau & pHKcl \\
\hline $\begin{array}{l}\text { Parcelle aménagée } \\
\text { en cordons } \\
\text { pierreux }\end{array}$ & Bande 1 & $58,49 \mathrm{a}$ & $7,5 \mathrm{a}$ & $0,69 \mathrm{a}$ & $6,4 \mathrm{a}$ & $5,02 \mathrm{a}$ \\
& Bande 2 & $67,38 \mathrm{a}$ & $7,3 \mathrm{a}$ & $0,64 \mathrm{a}$ & $6,35 \mathrm{a}$ & $4,74 \mathrm{~b}$ \\
Parcelle-témoin & Bande 3 & $63,13 \mathrm{a}$ & $5,4 \mathrm{a}$ & $0,52 \mathrm{a}$ & $6,27 \mathrm{a}$ & $4,56 \mathrm{~b}$ \\
\hline & Témoin & $28,82 \mathrm{~b}$ & $4,9 \mathrm{a}$ & $0,48 \mathrm{a}$ & $6,23 \mathrm{a}$ & $4,68 \mathrm{~b}$ \\
\hline & Pr $\square \mathrm{F}$ & 0,0001 & 0,08 & 0,483 & 0,467 & 0,001 \\
& Signif. & HS & NS & NS & NS & HS \\
\hline
\end{tabular}

NB : HS : Hautement significatif; S : Significatif; NS : Non Significatif; Signif. : Signification. Les moyennes suivies de la même lettre ne sont pas significativement différentes au seuil de 5\%. (Nombre d'échantillons par traitement : $\mathrm{n}=9$ ).

\section{DISCUSSION}

Impact de la technique des cordons pierreux sur la macrofaune du sol

Les résultats obtenus montrent que la pose des cordons pierreux entraîne une augmentation hautement significative $(\mathrm{P}<$ $0,01)$ des populations de macrofaune du sol. En effet, le nombre d'individus rencontré dans les parcelles aménagées est plus de six (06) fois plus élevé que celui de la parcelle témoin. Les cordons pierreux sont des barrières physiques destinées à freiner le ruissellement et à réduire l'érosion. Il s'en suit une augmentation de l'infiltration de l'eau dans le sol, et la sédimentation des particules (sables, terre fine, matière organique) à l'amont de la diguette (Serpentié et Lamachère 1988; David, 2003), créant alors une situation favorable aux développements de nombreux organismes du sol tels que les termites, vers de terre, fourmis, etc. Les cordons pierreux améliorent non seulement l'abondance numérique de la macrofaune, mais aussi, et de façon hautement significative $(\mathrm{P}<0,01)$, la diversité.

On note la présence dans la parcelle aménagée de populations de macrofaune appartenant aux familles de Formicidae, Lumbricidae, Termitidae, et considérées comme des ingénieurs du sol (Jones et al., 1994). La famille des termitidae, connue pour ses effets améliorants de la fertilité du sol, ne représente que $9 \%$ de la macrofaune recensé dans le champ témoin. En effet, les termites améliorent la structure physique des sols, grâce à l'enrichissement en éléments fins que 
réalisent les ouvriers, de sorte que l'horizon superficiel qui environne une termitière évolue vers une structure fine et dépourvue de graviers (Seugué, 2002). De plus, par la construction de leurs nids, leurs systèmes de galeries de récolte et par le brassage du sol remanié et apporté en surface à partir des horizons profonds du sol, les termites améliorent la stabilité structurale et la porosité du sol (Mando, 1997). L'indice de diversité de Shannon indique que la richesse spécifique est fonction de la pente. Sa valeur décroît en effet $\mathrm{du}$ haut de pente vers le bas de pente. Il est bien connu que les cordons pierreux entraînent une augmentation de la capacité d'infiltration de l'eau du sol (Zougmoré et al., 2000a). De même, l'excès d'eau détermine des pièges de tension superficielle et le danger des phénomènes d'endosmose ainsi que le manque possible d'air (Bachelier, 1978). Le degré de sensibilité étant fonction de l'espèce, il va alors s'installer une sélection qui réduira la richesse spécifique au fur et à mesure que l'on s'approche de la ligne de cordons pierreux. La répartition des groupes taxonomiques est aussi fonction de la réduction de la vitesse du ruissellement, et de la pente. En effet, l'indice d'équitabilité est nettement plus élevé dans le champ avec cordons pierreux, indiquant une meilleure répartition des espèces. On observe qu'il a tendance à décroître avec la pente, confirmant la perturbation de l'équilibre des populations due à l'augmentation de l'infiltration de l'eau dans le sol.

Impact de la technique des cordons pierreux sur les paramètres physicochimiques et l'activité microbienne du sol

L'amélioration des paramètres physiques du sol est due à l'accumulation des sédiments favorisée par la barrière physique que constituent les cordons pierreux (Hien, 1995, David, 2003, Ndiaye, 2009) et l'action de la macrofaune du sol. En effet, comme indiqué précédemment, la pose des cordons pierreux a entraîné une augmentation de la macrofaune du sol, en particulier des populations de Formicidae et de Termitidae, et ces dernières sont à l'origine d'apports de quantités plus ou moins importantes d'argiles pour la construction de leurs structures (Holt et Lepage, 2000).

Bationo et al. (1998) ont montré que les terres perdues du fait de l'érosion sont 2,5 fois plus riches en éléments nutritifs que le sol restant. On devrait alors s'attendre à une nette amélioration de la teneur du sol en carbone organique. Cependant, dans la présente étude, la pose de cordons pierreux n'a pas amélioré de façon significative la teneur du sol en carbone organique et en azote. Fondamentalement, la quantité de matière organique dans le sol est la résultante de deux processus antagonistes: la production primaire ou, plus exactement, la fraction de cette production qui rentre dans le sol, et la minéralisation microbienne, qui dépend de la qualité des MO incorporées et de l'intensité de l'activité microbienne (Nacro, 1997). Nos résultats ont montré que cette dernière est très élevée pour le champ aménagé en cordon pierreux, en particulier en haut de pente. Il en résulte donc une faible accumulation des composés organiques dans le sol. Autrement dit, l'efficacité des cordons pierreux pour le relèvement du statut organique du sol sera déterminée par son association à d'autres techniques culturales telles que l'apport de fumure organique. De plus, les deux années d'aménagement restent insuffisantes pour percevoir l'impact effectif sur les caractéristiques physico-chimiques. Des observations longues durées doivent être faites en vue de cerner davantage ces questions.

\section{Impact de la technique des cordons pierreux sur l'activité microbienne du sol}

Les communautés microbiennes des sols sont des maillons essentiels du fonctionnement des sols. Les résultats obtenus dans cette étude montrent que les plus forts potentiels respiratoires s'observent en haut et milieu de pente (Bande 1 et Bande 2). Les plus faibles quantités de $\mathrm{CO}_{2}$ dégagées sont notées au niveau du bas de pente et au niveau du témoin. La comparaison des résultats de la parcelle aménagée en cordons pierreux à ceux 
$\mathrm{du}$ témoin montre que le pourcentage d'éléments fins et la teneur en matières azotées sont les facteurs déterminant l'activité biologique. En effet, la macrofaune du sol participe non seulement à la minéralisation de l'azote organique par leur digestion, mais constituent aussi par eux-mêmes une réserve souvent importante et mobilisable à leur mort (Mathieu, 2004). Ainsi, la présence d'une faune plus abondante et diversifiée dans la parcelle aménagée en cordons pierreux pourrait expliquer la forte activité biologique au niveau de ce sol, et les meilleures croissances des plantes observées dans les champs aménagés avec les cordons pierreux (Yougbaré, 2008). L'activité biologique baisse au fur et à mesure que l'on se dirige vers le bas de pente, et se rapproche de celle du sol témoin malgré la présence des bandes de pierres. Cela pourrait s'expliquer par la baisse de l'aération du sol due certainement à une humidité beaucoup plus importante, inhibant ainsi l'activité microbienne.

\section{Conclusion}

L'étude s'est déroulée dans une zone marquée par une dégradation importante des sols et des ressources naturelles en général. Les résultats corroborent l'absence d'effet positif des cordons pierreux sur le stockage des composés organiques tel que révélés par les résultats de la littérature et indiquant que les cordons pierreux ne peuvent à eux seuls assurer la conservation de la fertilité du sol (Zougmoré et al., 2002). Toutefois, l'impact positif des cordons pierreux sur l'humidité du sol, l'atterrissement et le piégeage des particules solides en amont des cordons pierreux (Zougmoré et al., 2000) ont favorisé le développement d'une population importante de la macrofaune du sol, de même que l'accélération des processus de minéralisation de la matière organique du sol qui pourrait expliquer le faible stockage de la matière organique dans le sol. Les cordons pierreux pourraient donc entraîner un appauvrissement du sol en composés organiques, si la technique n'est pas accompagnée d'un apport de fumier ou de compost dans les différentes bandes de cordons de pierre. Cela peut se faire via la pratique combinée de la technique des cordons pierreux avec d'autres pratiques culturales utilisées pour la réhabilitation des sols dégradés (demi lune, zaï, association de cultures, etc.). L'apport de substrats organiques compensera alors les pertes dues au lessivage et au prélèvement par les cultures. L'association de la fumure organique est donc nécessaire dans les parcelles aménagées en cordons pierreux pour une gestion durable de la fertilité des sols.

\section{REMERCIEMENTS}

Ce travail a été réalisé dans le cadre de suivi de l'impact du projet SILEM sous la coordination de l'UICN au Burkina Faso. Que cette institution soit remerciée, en particulier le chef de Mission, le Professeur Aimée NIANOGO. Nous voudrions également témoigner notre reconnaissance à Madame HONADIA Clarisse, à tout le personnel du SILEM et aux populations du village de Kampelzougou pour les facilités offertes lors de nos campagnes de terrain.

\section{REFERENCES}

Anderson JM, Ingram JS. 1989. Tropical Soil Biology. Handbook of Methods. CAB International: Suxes; $117 \mathrm{p}$.

Bachelier G. 1978. La faune des sols, son écologie et son action. Initiation Documentations Techniques $\mathrm{n}^{\circ} 38$. ORSTOM, Paris, 391p.

Bachelier G. 1973. Activité biologique et techniques simples qui permettent l'évaluation. Cah .ORSTOM, série 77.

Bationo A, Kihara J, Vanlauwe B, Waswa B, Kimetu J. 2007. Soil organic carbon dynamics, functions and management in West African agro-ecosystems. Agricultural Systems, 94: 13-25.

Bationo A, Lompo FS, Koala S. 1998. Research on nutrient flows and balances in West Africa: state-of-the-art. Agric. Ecosyst. Envir., 71: 19-35.

Bland RG, Jacques HE. 1987. How to Know the Insects. The Pictured Key Nature Series K.W.C. (3rd edition). Brown Company Publishers: Dubuque, Lowa, USA; 409p. 
Bremner JM. 1965. Inorganic Forms of Nitrogen. In Methods of Soils Analysis. Part 2. Black CA (ed). Am. Soc. Agron.: Madison, USA; 1179 - 1237.

CIRAD, CNEARC. 1998. Reconnaissance des hyménoptères parasitoïdes d'importance économique, clé Iconographique pour l'identification des genres. Cotonou, Bénin. 321p.

David F. 2003. Action de la macrofaune sur la fertilité des sols dans une succession végétale: rôle des termites dans un système de zaï forestier au Yatenga (Burkina Faso). Mémoire d'IngénieurMaître, Institut Universitaire Professionnalisé Environnement, Technologies et Société, 25p.

Delvare G, Aberlenc H. 1999. Les Insectes d'Afrique et d'Amérique Tropicale. Glecs pour la Recommandation des Familles. Imprimerie Caballery-58500 Clamecy, France; 302p.

Doamba WSMF. 2007. Effet du zaï forestier sur l'évolution de la biodiversité et des paramètres physiques, chimiques et biologiques du sol. Mémoire de fin de cycle IDR/UPB. $111 \mathrm{p}$

Dommergues Y. 1960. La notion de coefficient de minéralisation du carbone dans les sols. Agron. Trop., 15 : 5460.

Duchaufour PH. 1970. Précis de Pédologie (3ème édition). Masson (ed) : Paris ; 481p.

Dugué P, Roose E, Rodriguez I. 1993. L'aménagement de terroirs villageois et l'amélioration de la production agricole au Yatenga (Burkina Faso): Une expérience de recherche-développement. Cah. Orstom, sér. Pédol., 28(2): 385-402.

Feller C. 1979. Une méthode de fractionnement granulométrique de la matière organique des sols. Application aux sols tropicaux, à textures grossières, très pauvres en humus. Cah. ORSTOM, sér. Pedol., 17(4): 339-346.

Fontes J, Guinko S. 1995. Carte de la végétation et l'occupation du sol du Burkina Faso. Ministère de la coopération française. Projet Campus, $66 \mathrm{p}$.

Henao J, Baanante CA. 2006. Agricultural production and soil nutrient mining in Africa: Implications for resource conservation and policy development. Technical bulletin T-72, International
Fertilizer Development center (IFDC), Muscle shoals, Al.

Hien FG. 1995. La régénération de l'espace sylvo-pastoral au sahel: une étude de l'effet de mesures de conservation des eaux et des sols au Burkina Faso. Thèse de l'université Agronomique Wageningen, 219p.

Holt JA, lepage M. 2000. Termite and soil properties. In Termites: Evolution, sociality, Symbiosis, Ecology. Abe T, Bignell DE, Higashi M. Kluwer Academic Publishers: Dordrecht; 389407.

INERA. 2000. Rapport sur les acquis scientifiques 1992-1999 du département gestion des ressources naturelles et systèmes de productions. (GRN/SP)/ Ouagadougou, 139p.

Jones CG, Lawton JH, Shachak KM. 1994. Organisms as ecosystem engineers. Oikos, 69(3): 373-86.

Kambou FN, Zougmoré R. 1995. Evolution des états de surface d'un zipellé soumis à différentes techniques de restauration des sols? Yilou, Burkina Faso. 12 ème journée du Reseau Erosion, Paris 14p.

Katyal JC, Vlek PLG. 2000. Desertification concept, causes and amelioration ZEFDiscussion Papers $N^{\circ} 33$ on Development Policy. Bonn, October 2000. Center for Development Reseach (ZEF). Bonn, Germany. 65pp.

Mando A. 1997. The effect of termites and mulch on the water balance of Sahelian crusted-soils. Soil Technology, 11: 121138.

Mathieu J. 2004. Etude de la macrofaune du sol dans une zone de déforestation en amazonie du Sud-Est, au brésil, dans le contexte de l'Agriculture familiale. Thèse de doctorat/Université Pierre et Marie Curie, Paris 6, 221p.

Nacro HB. 1997. Hétérogénéité de la matière organique dans un sol de savane humide (Lamto, Côte d'Ivoire): caractérisation chimique et étude in vitro, des activités microbiennes de minéralisation du carbone et de l'azote, Thèse de Doctorat de l'Université, Paris 6, 302p.

Ndiaye S. 2009. Evaluation de l'impact des cordons pierreux et de la fertilité des sols dans deux microprojets finances par le 
CILSS au Sénégal. Rapport d'étude, GREEN/CILSS, $30 \mathrm{p}$.

Pieri C. 1989. Fertilité des terres de savanes. Bilan de trente ans de recherche et de développement agricoles au Sud du Dahara. Ministère de la coopération.444p.

Roose E, Dugué P, Rodriguez L. 1993. L'aménagement des terroirs villageois et l'amélioration de la production agricole au Yatenga (Burkina Faso). Une expérience de recherche-développement. Cahiers ORSTOM, Série Pédologique, 27(2): 385- 402.

Saidani S. 1995. L'évaluation de l'impact des termites du genre Cubitermes sur la conservation du stock organique de parcelles cultivées de la vallée du Niari (République du Congo) DESS. Université Paris XII Val De marne. 63p.

Sangaré S. 2002. Evaluation des performances agro-écologiques des techniques de lutte contre la désertification dans les provinces du Passoré et du Yatenga. Cas du zaï, de la demi-lune et du tapis herbacé. Mémoire de fin d'études, Université Polytechnique de BoboDioulasso-Institut du Développement Rural (UPB-IDR), 85p.

Scherr SJ. 1999. Soil degradation, a threat to developing-country food security by 2020? Food, Agriculture, and the Environmental Discussion. Paper 27. International Food Policy Research institute, Washington, DC.

Serpentié G, Lamachère JM. 1988. Valorisation des eaux de ruissellement en zone soudano-sahélienne. Burkina Faso, Province du Yatenga, Région du Bidi. ORSTOM, Ouagadougou. 19p.

Seugué C. 2002. Les structures biogéniques de termites et de vers de terre dans un écosystème sub-saharien: quantification et typologie. Thèse de Doctorat Université Paris XII-VAL DE MARNE, $130 \mathrm{p}$.

Stroorvagel JJ, Smaling EMA. 1990. Assessment of soil nutrient depletion in sub-sahara Africa. 1983-2000, vol 1- 4Report $\mathrm{n}^{\circ} 28$, vol. 2. Winand staring centre, Wageningen.

Tiessen H, Moir IO. 1993. Total and organic carbon. In Soil Sampling and Methods of Analysis, Carter MR (ed). Can. Soc. Sol Sci.: Lewis; 187-199.

Villiers A. 1997. Hemiptères de Ramées Boubee: Paris, France; 301p.

Walkley A, Black IA. 1934. An examination method of the detjareff and a proposed modification of the chromic acid titration method. Soil Science, 37: 29-38.

Yougbaré H. 2008. Evaluation de la fertilité des terres aménagées en cordons pierreux, zaï et demi-lunes dans le bassin versant du Zondoma. Mémoire d'Ingénieur, IDR/UPB, 55 p.

Zougmoré R, Guillobez S, Kambou FN, Son G. 2000a. Runoff and sorghum performance as affected by the spacing of stone lines in the semiarid sahelian zone. Soil and Tillage Research, 56: 175183.

Zougmoré R, Kaboré D, Lowenberg-Deboer J. 2000b. Optimal spacing of soil conservation barriers: example of rock bunds in Burkina Faso. Agronomy Journal, 92: 361-368.

Zougmoré R, Gnankambary Z, Guillobez S, Stroosnijder L. 2002. Effect of stone lines on soil chemical characteristics under continuous sorghum cropping in semi-arid Burkina Faso. Soil and Tillage Research, 66: 47-53. 